\title{
O EFEITO QUE AS COMUNIDADES DE MARCA GERAM NO AMOR À MARCA
}

\section{THE EFFECT BRAND COMMUNITIES GENERATE IN BRAND LOVE}

\section{RESUMO}

Este artigo tem como objetivo identificar os impactos das comunidades de marcas em constructos relacionais, como passa-a-palavra, advocacia e lealdade, por meio dos efeitos do amor à marca. Foram recolhidos 510 questionários válidos entre os consumidores portugueses. A modelagem de equações estruturais foi utilizada para testar as hipóteses propostas. Há duas importantes contribuições: investigar os impactos das comunidades de marca e usar os efeitos mediadores do amor à marca nos resultados relacionais. Essa investigação mostra como as comunidades de marca podem contribuir para reforçar os laços entre marcas e clientes, introduzindo o amor nesses relacionamentos.

Palavras-chave: Comunidades de marca. Amor à marca. Lealdade. Advocacia. Passa-a-palavra.

\begin{abstract}
This article aims to identify the impacts of brand communities on relational constructs such as word-of-mouth, advocacy and loyalty through the effects of brand love. 510 valid questionnaires were collected from Portuguese consumers. Structural equation modeling was used to test the proposed hypotheses. We have two important contributions: investigating the impacts of brand communities and using the mediating effects of brand love on relational outcomes. This research shows how brand communities can contribute to strengthening ties between brands and customers by introducing love into those relationships.
\end{abstract}

Keywords: Brand communities. Brand love. Loyalty. Advocacy. Word of mouth.

Filipa Antunes Peres filipaantunesperes@gmail.com Licenciada em Design de Moda e Têxtil e Mestre em Marketing pela Faculdade de Economia da Universidade de Coimbra.

\section{INTRODUÇÃO}

O marketing relacional é uma das ferramentas que procura criar e manter relacionamentos de longo prazo com seus consu- 
midores e é visto como um recurso estratégico para uma empresa, uma tendência para o marketing moderno e relacionamentos bem-sucedidos (WEBSTER, 1992; MORGAN; HUNT, 1994; MATTSSON, 1997; GUMMESSON, 2002; LAROCHE et al., 2012). As comunidades de marcas estão a ser vistas como uma forte ferramenta para reforçar essas relações. Elas realizam importantes tarefas da marca, compartilhando informações, perpetuando uma história e cultura de marca e prestando atendimento ao cliente (MUNIZ JUNIOR; O'GUINN, 2001). Uma comunidade de marca é definida como uma comunidade especializada, baseada em um conjunto estruturado de relações sociais entre os admiradores de uma marca (MUNIZ JUNIOR; O'GUINN, 2001). Essas comunidades são fundadas com o objetivo de conectar não apenas clientes entre si, mas também uma marca / empresa a um cliente próprio. Comunidades de marca são essenciais para criar relacionamentos duradouros e significativos com os indivíduos (MCALEXANDER; SCHOUTEN; KOENIG, 2002). Para um cliente sentir a marca, é necessário saber o que está a acontecer com ela. Mais especificamente, um amor à marca pode surgir, definindo-se como o grau emocional e apaixonado de afeto que um consumidor satisfeito tem em relação a uma marca em particular (AHUVIA, 2005). O principal objetivo deste estudo é entender como as comunidades de marcas podem contribuir para estabelecer relações duradouras com os clientes, nomeadamente, por meio da lealdade, advocacia e WOM, com base no papel mediador do amor à marca. A fim de encontrar respostas para esses objetivos, foi realizado um estudo transversal, baseado em um questionário estruturado com 510 indivíduos. A modelagem de equações estruturais foi utilizada para testar as hipóteses desenvolvidas nessa investigação, para atingir os objetivos propostos. Essa investigação inova, estabelecendo uma relação empírica entre as comunidades de marca e o amor à marca, que medeia as relações entre as comunidades de marca e os resultados relacionais.

\section{HIPÓTESES DE DESENVOLVIMENTO E PESQUISA CONCETUAL}

\subsection{COMUNIDADE DA MARCA}

Uma comunidade é um lugar de conforto e aconchego, em que todos os elementos alcançam um entendimento mútuo entre as partes (BAUMAN, 2003). As comunidades podem ser definidas como um lugar no qual os membros podem discutir amistosos sobre todos os temas; em que a ajuda mútua não é apenas uma obrigação, mas o membro também pode esperar ajuda e apoio quando necessário (BAUMAN, 2003). Muniz Junior e O'Guinn (2001, p. 412) definem a comunidade de marcas como "uma comunidade especializada, não geograficamente ligada, baseada em um conjunto estruturado de relações sociais entre os usuários de uma marca." As comunidades de marca são compostas de pessoas que possuem uma identificação social com outras pessoas, que compartilham seu interesse em uma determinada marca (ALGESHEIMER; DHOLAKIA; HERRMANN, 2005; MCALEXANDER; SCHOUTEN; KOENIG, 2002). Esses tipos de comunidades apresentam-se como especializadas porque, e nas palavras de McAlexander, Schouten e Koenig (2002, p. 38), "no seu centro, a comunidade é caracterizada por uma consciência compartilhada entre os membros da comunidade." Segundo Muniz Junior e O'Guinn (2001, p. 412), essas comunidades têm a particularidade de se basear "na consciência compartilhada de vários tipos de rituais e tradições e responsabilidade moral e obrigações para com a sociedade". Atualmente, os consumidores são altamente exigentes com as marcas de que gostam, e em um cenário de crise econômica, a criação de uma comunidade de marca pode ser de grande importância para a gestão da marca (MILLÁN; DÍAZ, 2014; KUENZEL; HALLIDAY, 2008). Em geral, as empresas hoje estão a ver as marcas como veículos para fazer e construir fortes conexões emocionais com seus clientes e, portanto, que podem gerar fidelidade e lucrati- 
vidade (HUNG, 2014; PARK et al., 2009). Os investigadores da marca têm-se interessado em analisar os mecanismos de formação das marcas dos consumidores (HUNG, 2014; PARK; MACINNIS, 2006; THOMPSON; SINHA, 2008). Ao referir-se às comunidades de marcas, os investigadores descobriram que a identificação com a marca é um estímulo crítico e significativo para a participação da marca comunitária (HUNG, 2014; BAGOZZI; DHOLAKIAM, 2002; DHOLAKIA; BAGOZZIA; PEARO, 2004). Nos trabalhos de de Dholakia, Bagozzia e Pearo (2004), níveis mais altos de valor percebido levam a uma identificação mais forte da comunidade, mas os mecanismos que levam à identificação permanecem obscuros. No entanto, acredita-se que os membros da comunidade gritam por autenticidade e reconhecimento entre a comunidade, e eles podem levar à autoidentificação com o grupo. Essa identificação e autoexpressão autênticas funcionam como um processo de identificação (HUNG, 2014; CABLE; GINO; STAATS, 2013). As comunidades de marcas têm duas dimensões principais diferentes (ZHOU et al., 2012): 1 . Identificação com a comunidade de marca; 2. Compromisso com a comunidade de marca. Ao falar sobre a identificação com a comunidade da marca, Dholakia, Bagozzia e Pearo (2004) argumentam que a identificação relaciona-se com as avaliações individuais de pertencer a uma comunidade de marca. Isso significa que o indivíduo experimentará um apego ou um compromisso afetivo com a comunidade e com a própria marca (BAGOZZI; DHOLAKIA, 2002; ELLEMERS et al., 1999). A identificação com a comunidade da marca refere-se ao autoconceito de uma pessoa que a torna igual a outros membros do grupo e distinta de pessoas de fora. Esse tipo de definição e identificação permite que uma pessoa participe ativamente como membro da comunidade da marca, bem como mantenha relações positivas com os outros membros (HUNG, 2014; TSAI; HUANG.; CHIU, 2012; BHATTACHARYA; SEN, 2003; BERGAMI; BAGOZZI, 2000). Fica claro que indivíduos que têm um interesse comum e uma alta identificação de marca participarão ativamente da comunidade da marca (SHAARI; AHMAD, 2016). A identificação pode levar ao comprometimento e ao compromisso com a comunidade de marca, que é definido como um apego psicológico a uma comunidade e à sua crença no valor do relacionamento (KIM et al., 2008). Um relacionamento de marca e compromisso de longo prazo desenvolver-se-ão quando os indivíduos acreditarem que a marca reflete a sua personalidade e aumenta a sua autoestima e status social (ZHOU et al., 2012; WANG, 2002). Os consumidores tendem a comprar a mesma marca de forma consistente, criando um relacionamento único, semelhante ao amor (ZHOU et al., 2012; KIM et al., 2008; ALGESHEIMER; DHOLAKIA; HERRMANN, 2005). Para Jang et al. (2008), a participação comprometida e as interações com outros membros da comunidade ajudam a fortalecer a experiência e o valor da marca dos consumidores, levando a um maior comprometimento da marca e da comunidade, além de uma maior lealdade à marca e à comunidade. As comunidades de marca têm um papel muito importante quando se trata de amor à marca, na medida e no elogio para estabelecer uma conexão mais forte com ela. Esta pesquisa também mostra relacionamentos consolidados que vêm do amor à marca, como o passa-palavra, a advocacia da marca e a fidelidade à marca. Autores como Zhou et al. (2012), Kim et al. (2008) e Algesheimer, Dholakia e Herrmann (2005) desenvolveram pesquisas prévias em que podem relacionar a conceção de marca comunitária com a conceção de amor à marca. Além disso, autores como Morgan e Hunt (1994) e McAlexander, Schouten e Koenig (2002) realizaram estudos que refletem que o passa-a-palavra, a advocacia da marca e a fidelidade à marca nascem como resultado de comunidades de marca quando apresentadas como resultantes delas.

\subsection{OS ANTECEDENTES DAS CO- MUNIDADES DA MARCA}

A seguir, serão apresentados alguns dos 
antecedentes que diversos estudos consideram relevantes para a explicação e a perceção do tema principal deste trabalho, Comunidades de Marca.

\subsubsection{Necessidade de afiliação}

Os autores Baumeister e Leary (1995) entendem a necessidade de afiliação como um atributo da personalidade humana. É um desejo que os indivíduos têm de manter o contato social, bem como suas tendências de receber recompensas sociais por meio de relações harmoniosas e agradáveis com outros seres humanos e comunidades (TSAI; HUANG; CHIU, 2012). O humano precisa se sentir amado e sentir que também ama (HILL, 1987). Isso sugere que sentimentos de solidão podem ser precipitados, tanto por falta de contato social, quanto por falta de um relacionamento mais íntimo com outros grupos ou comunidades (WEISS, 1973; SHAVER; BUHRMESTER, 1983). Autores como McAlexander, Schouten e Koenig, (2002) e Wasko et al. (2004) realizaram estudos que mostram que essa variável tem impacto positivo nas comunidades de marca quando apresentada como sua antecedente.

Hipóteses de investigação que são formuladas

H1a: existe uma relação positiva entre Necessidade de Afiliação e Comunidade de Marca (Dimensão Identificação com a Comunidade da Marca).

H1b: existe uma relação positiva entre Necessidade de Afiliação e Comunidade de Marca (Dimensão Compromisso com a Comunidade da Marca).

\subsubsection{Identificação}

A variável identificação está relacionada com o conceito que o indivíduo possui de si de acordo com as definições e as características que definem a pessoa em uma determinada categoria social. Também são ponderadas as medidas que tornam o assunto imutável no que toca ao estereótipo individual como pes- soa, bem como a seu grupo, bem como a outros para o grupo (BERGAMI; BAGOZZI, 2000; BHATTACHARYA; SEN, 2003). Ao criar ligações, o indivíduo terá, com os outros participantes da comunidade, uma conformidade com seus ideais, pensando nos membros da comunidade de maneira semelhante. Isso garante que a identificação seja construída por princípios de afiliação e pertença (BAGOZZI; DHOLAKIA, 2002). Autores como Algesheimer, Dholakia e Herrmann (2005), McAlexander, Schouten e Koenig (2002) e Wasko et al. (2004) realizaram estudos que sustentam que a identificação tem impacto positivo nas comunidades de marcas quando apresentadas como seus antecedentes.

Hipóteses de investigação que são formuladas

H2a: existe uma relação positiva entre a Identificação e a Comunidade de Marca

(Dimensão Identificação com a Comunidade da Marca).

H2b: existe uma relação positiva entre Identificação e Comunidade de Marca

(Dimensão Compromisso com a comunidade da marca).

\subsubsection{Extroversão}

A extroversão está ligada a experiências emocionais e desempenhos cognitivos (TAMIR, 2005). Essa variável é representada como uma tendência a ser mais sociável, assim como uma maior tendência a experimentar sentimentos mais positivos (LUCAS et al., 2000). De acordo com Gray (1970), existem três sistemas de motivações nos humanos que os fazem esperar por uma recompensa de outros indivíduos, trabalhando a extroversão como uma ponte entre o indivíduo e os grupos ou comunidades. Autores como McAlexander, Schouten e Koenig (2002) e Wasko et al. (2004) realizaram estudos em que a extroversão tem impacto positivo nas comunidades de marca quando apresentada como seu antecedente.

Hipóteses de investigação que são formuladas

H3a: existe uma relação positiva entre 
Extroversão e a Comunidade de Marca

(Dimensão Identificação com a Comunidade da Marca).

H3b: existe uma relação positiva entre Extroversão e a Comunidade de Marca

(Dimensão Compromisso com a comunidade da marca).

\subsubsection{Satisfação}

Para Tse e Wilton (1988), a satisfação pode ser entendida como uma resposta do cliente para avaliar a diferença entre suas expectativas anteriores e o desempenho real do produto/marca, percebido após o consumo desse produto/marca. É ainda definido como um sentimento de prazer que o indivíduo recebe, ao consumir um produto, e preenche a sua necessidade, meta, desejo, ou outros (OLIVER, 1997). Satisfação requer lealdade, o que significa que, quando um indivíduo está satisfeito, o seu passa-a-palavra seja talvez também mais forte, quando combinado com comunidades de marca ou grupos que apoiam a mesma marca simultaneamente (MITTAL; KAMAKURA, 2001). Hipóteses de investigação que são formuladas.

H4a: existe uma relação positiva entre Satisfação e Comunidade de Marca

(Dimensão Identificação com a Comunidade da Marca).

H4b: existe uma relação positiva entre Satisfação e Comunidade de Marca

(Dimensão Compromisso com a comunidade da marca).

\subsubsection{Identificação com a marca}

A Identificação com a Marca é definida por Escalas e Bettman (2005) como o grau de integração de uma marca ao próprio conceito do indivíduo. Quando se percebe um alto nível de identificação com a marca, o indivíduo sente que ela é congruente com sua própria imagem (SIRGY, 1982). Onde um ou mais indivíduos compartilham características que os distinguem da multidão, eles sentir-se-ão emocionalmente mais envolvidos um com o outro, sentindo um maior nível de identificação não apenas pessoalmente, mas também socialmente, criando um novo autoconceito próprio (HOGG, 2006; KUENZEL; HALLIDAY, 2008). Autores como Jones e Kim (2011) e McAlexander, Schouten e Koenig (2002) realizaram estudos que demonstram como a identificação da marca tem influência positiva quando apresentada como sua antecedente.

Hipótese de investigação que é formulada

H5a: existe uma relação positiva entre a Identificação com a Marca e a Comunidade de Marca (Dimensão Identificação com a Comunidade da Marca).

H5b: existe uma relação positiva entre a Identificação com a Marca e a Comunidade de Marca (Dimensão Compromisso com a Comunidade da Marca).

\subsubsection{Confiança da marca}

A Confiança da Marca é descrita com a disposição do consumidor médio para ver qual a capacidade da marca para executar a sua função declarada (CHAUDHURI; HOLBROOK, 2001; SCHAU; MUNIZ; ARNOULD, 2009). É um ingrediente-chave para o desenvolvimento de relações de marca (MORGAN; HUNT, 1994; CARROLL; AHUVIA, 2006; MCALEXANDER; SCHOUTEN; KOENIG, 2002). A confiança precisa ser construída, de modo que as partes possam confiar umas nas outras (ALTMAN; TAYLOR, 1973; CHAUDHURI; HOLBROOK, 2001). Autores como Morgan e Hunt (1994) e McAlexander, Schouten e Koenig (2002) realizaram estudos que mostram que a confiança da marca tem um efeito positivo nas comunidades de marca, quando apresentada como sua antecedente.

Hipótese de investigação que é formulada

H6a: existe uma relação positiva entre Confiança da Marca e a Comunidade de Marca (Dimensão Identificação com a Comunidade da Marca) 
H6b: existe uma relação positiva entre Confiança da Marca e a Comunidade de Marca (Dimensão Compromisso com a Comunidade da Marca).

\subsubsection{Satisfação com o relacionamento}

A satisfação que o consumidor tem com a sua relação com a marca é considerada o estado afetivo do consumidor, resultante de uma apreciação global de sua relação com a marca (JAP, 2011). Uma relação satisfatória com ela pode levar o indivíduo a procurar e interagir com consumidores que pensem de maneira semelhante e que compartilhem seu entusiasmo (ALGESHEIMER; DHOLAKIA; HERRMANN 2005). Muñiz Junior e O'Guinn (2001), no seu estudo de comunidades de marca, utilizando dados etnográficos, conseguiram entender o nível de relacionamento que o consumidor tem com uma marca ao especificar quais marcas são preferidas por ele e pesquisar as comunidades de marca. Estudos realizados pelos autores Muñiz Junior e O'Guinn (2001), McAlexander, Schouten e Koenig (2002) e Wasko et al. (2004) refletem que essa variável tem um impacto positivo nas comunidades de marca, quando apresentada como antecedente. Hipóteses de investigação que são formuladas.

H7a: existe uma relação positiva entre Satisfação com o relacionamento e

Comunidade de Marca (Dimensão Identificação com a Comunidade da Marca).

H7b: existe uma relação positiva entre Satisfação com o relacionamento e

Comunidade de Marca (Dimensão Compromisso com a Comunidade da Marca).

\subsubsection{Prestígio da marca}

A variável Prestígio da Marca refere-se ao estado de posicionamento de um produto associado a uma marca (MCCARTHY; PERREAULT, 1987; STEENKAMP et al., 2003). Uma imagem de marca de prestígio pode induzir o sentimento psicológico da experiência e per- tencer a classes sociais mais altas, melhorando o autoconceito do indivíduo (STEENKAMP et al., 2003; SWEENEY; SOUTAR, 2001). Indivíduos, quando inseridos em uma sociedade, grupo ou comunidade, são mais influenciados a consumir essa marca. É mais fácil para um comprador ser tentado a comprar o prestígio, impulsionado pela ideia de que, se o resto do grupo compra essa marca, o eu também deve comprar.

Hipóteses de investigação que são formuladas

H8a: existe uma relação positiva entre Prestígio da Marca e Comunidade da Marca (Dimensão Identificação com a Comunidade da Marca).

H8b: existe uma relação positiva entre Prestígio da Marca e Comunidade da Marca (Dimensão Compromisso com a Comunidade da Marca).

\subsubsection{Identidade da marca}

A Identidade da Marca é a ideia única e essencial para ela, sugerindo que a marca é a memória de um produto e deve agir como referência longa e resistente (AAKER, 1996; CHERNATONY, 2010; KAPFERER, 2008). Uma marca deve ser capaz de suportar tempos de mudança, e é possível que ela possa mudar a sua identidade em circunstâncias excepcionais, embora ela deva manter a sua identidade por longos períodos de tempo, resistindo à tentação de mudar de identidade (AAKER, 1996; KAPFERER, 2008), tornando, assim, mais fácil a marca da comunidade e também manter sua identidade, seja social ou geral.

Hipóteses de investigação que são formuladas

H9a: existe uma relação positiva entre a Identidade da Marca e a Comunidade da Marca (Dimensão Identificação com a Comunidade da Marca).

H9b: existe uma relação positiva entre a Identidade da Marca e a Comunidade da Marca (Dimensão Compromisso com a Comunidade da Marca). 


\subsubsection{Afeto da marca}

O Afeto da Marca é traduzido com o potencial que ela tem para induzir uma resposta emocional positiva do consumidor ao resultado de seu uso (CHAUDHURI; HOLBROOK, 2001). A literatura sobre a representação de episódios mentais demonstra que as representações mentais podem ser episódios afetivos e estimulantes (BOWER; FORGAS, 2000). Quando há afeto material, é certo que os consumidores são os avaliadores de uma marca e podem ser influenciados pelo ambiente onde estão ou pela pertença a um grupo ou comunidade, o que faz que tenham respostas emocionais diferentes daquelas que teriam se não fossem inseridos nesses grupos (KEMPF, 1999; KIM; MORRIS, 2007). Autores como Muniz Junior e O'Guinn (2001), McAlexander, Schouten e Koenig (2002) e Scarpi (2010) realizaram estudos que sustentam que o Brand Affect tem impacto positivo nas comunidades de marca quando apresentado como antecedente de si. Hipóteses de investigação que são formuladas.

H10a: existe uma relação positiva entre Afeto da Marca e a Comunidade de Marca

(Dimensão Identificação com a Comunidade da Marca).

H10b: existe uma relação positiva entre Afeto da Marca e a Comunidade da Marca

(Dimensão Compromisso com a Comunidade da Marca).

\subsection{CONSEQUENTES DAS COMUNI- DADES DA MARCA}

Aqui, serão apresentados alguns dos consequentes que diversos estudos consideram relevantes para a explicação e a perceção do tema principal deste trabalho, Comunidades de Marca.

\subsubsection{Amor à marca}

$\mathrm{O}$ amor à marca é definido como "o grau de afeição afetiva e emocional que um consumidor satisfeito tem com uma determinada marca." (CARROL; AHUVIA, 2006, p. 31). Com base na literatura sobre o conceito de amor-próprio, o amor à marca inclui paixão por ela, apego a ela, sua avaliação positiva, emoções positivas em resposta a ela e declarações de amor a ela (BELK, 1964). Os autores Carroll e Ahuvia (2006) diferenciam o amor à marca da satisfação e apego da marca. $\mathrm{O}$ amor à marca também pode ser caracterizado como íntimo e excitante, em que há um alto nível de compromisso entre a marca e o consumidor. Especificamente, esse amor é um pilar tridimensional que pode ser facilmente medido por variáveis como intimidade, paixão e comprometimento (KEH et al., 2007). Consequentemente, de acordo com a literatura recente (BATRA et al., 2012; FOURNIER, 1998), marcas de amor são superiores a outras marcas em uma ou mais qualidades. $\mathrm{O}$ amor à marca pode ser visto como o que comumente chamamos de "fanatismo" por uma marca. Os consumidores serão tão afetados por esse amor, que deixarão de consumir o que outras marcas podem oferecer. Esse fenômeno pode ser explicado pelos efeitos introduzidos pelas comunidades de marca, quando os participantes oferecem restrições para encerrar um relacionamento. Esse fenômeno dá asas à organização: é levado em consideração que o amor à marca aumenta a fidelidade à marca dos clientes, e as comunidades de marca aumentam tanto o amor quanto a lealdade. Isso traduz-se em uma atitude diferente em relação à marca. Consumidores que sintam amor por uma marca avaliarão o que é mais benéfico para eles, geralmente escolhendo uma marca favorita (CARROLL; AHUVIA, 2006; KEH et al., 2007). O consumidor pode querer adquirir uma marca, mas, por sentimentos de pertença, ele adquirirá uma que a comunidade elogie. Isso pode levar a um tipo de conflito emocional, pois é sabido que as comunidades podem tornar-se "anti-marcas" (MUNIZ JUNIOR; O'GUINN, 2001). Assim, o indivíduo que já gosta da marca, agora vai começar a amá-la, porque há membros da comunida- 
de que o encorajarão a nutrir o seu amor por ela (ALGESHEIMER; DHOLAKIA; HERRMANN, 2005; MUNIZ JUNIOR; O'GUINN, 2001). Quando um indivíduo experimenta um relacionamento de longo prazo com uma comunidade de marca, seus membros interagem diariamente entre si, tornando o valor associado a essa marca ainda maior dentro da comunidade (CARROLL; AHUVIA, 2006; PARK; MACINNIS, 2006).

\section{Hipóteses são propostas}

H11a: existe uma relação positiva entre Comunidade de Marca (Dimensão Identificação) e Amor à Marca.

H11b: existe uma relação positiva entre Comunidade de Marca (Dimensão Compromisso) e Amor à Marca.

\subsubsection{WOM (Passa-a-Palavra)}

Uma das definições primárias do Passa-a-Palavra é que fala sobre produtos e serviços entre pessoas à parte da propaganda de produtos ou serviços das empresas (MAISAM; MAHSA, 2016). Segundo os autores, essas discussões podem ser conversas mútuas ou recomendações e sugestões unilaterais. Um dos pontos cruciais ocorre nessas conversas por pessoas que têm um tempo muito curto para persuadir outras pessoas a usar esse produto (MAISAM; MAHSA, 2016). O Passa-a-Palavra também é definido como comunicação oral, pessoa a pessoa, entre um comunicador e um recetor, em que o recetor percebe a informação como não comercial sobre uma marca, produto ou serviço (ARNDT, 1967; BUTTLE , 1998). Também é definido por Batra et al. (2012, p. 2) como uma força para resistir a informações negativas que podem ser ditas contra a marca. Essa variável é, por vezes, considerada como publicidade gratuita que pode ser vista como qualquer forma paga de apresentação não pessoal de ideias, bens ou serviços por um patrocinador identificado (ALEXANDER, 1964). A WOM pode ser mais influente na mente do consumidor do que anúncios estáticos ou comerciais. Por meio do seu de- sempenho espontâneo e dinâmico, pode interagir com a mente do consumidor, mediante a consciência, as expectativas, as perceções, as atitudes e as intenções (HERR et al., 1991). WOM provou ser mais eficaz para aumentar a conscientização sobre os indivíduos e o teste do produto, por si só (SHETH, 1971). As Comunidades de Marca podem ser vistas como um lugar em que os membros podem discutir amistosos sobre todos os temas e onde podem encontrar ajuda e apoio mútuos quando precisam (BAUMAN, 2003). As Comunidades de Marca podem basear esse compromisso em conexões afetivas e emocionais com a marca e com a organização com a qual elas se envolvem tanto, que gostam de ser membros da organização (MAISAM; MAHSA, 2016; ZIAULLAH et al., 2015). As comunidades de marca compartilham valores e princípios, também congruência, o que leva a um maior comprometimento entre os membros, conversando entre eles sobre a marca e a organização, criando um boca-a-boca positivo (MORGAN; HUNT, 1994; MCALEXANDER; SCHOUTEN; KOENIG, 2002; LARASATI; HANANTO, 2012, MAISAM; MAHSA, 2016). Portanto, propomos as seguintes hipóteses.

H12a: existe uma relação positiva entre Comunidade de Marca (Dimensão Identificação) e Passa-a-Palavra.

H1b: existe uma relação positiva entre Comunidade de Marca (Dimensão Compromisso) e Passa-a-Palavra.

H15: existe uma relação positiva entre Amor à Marca e Passa-a-Palavra.

\subsubsection{Advocacia de marca}

A advocacia da marca pode ser vista como uma promoção ou defesa de uma organização, um produto ou uma marca, de um consumidor para outro, e o nível máximo de aprovação da relação entre o consumidor e a marca (WALZ; CELUCH, 2010). Advocacia é uma forma de orientação para o mercado, conhecimento e envolvimento do consumidor, no caso de uma busca por um relacionamento in- 
tenso com níveis máximos de confiança, como uma parceria (LAWER; KNOX, 2006). Por meio de comunidades de marca, os consumidores podem defender e preservar a marca, tornando-se mais conscientes dos esforços que a organização faz para a satisfação dos clientes.A advocacia da marca é uma forma avançada de orientação para o mercado, que considera as preocupações, o conhecimento e o envolvimento de consumidores que fazem parte não apenas da comunidade da marca, mas da marca globalmente (LAWER; KNOW, 2006; CARROLL; AHUVIA, 2006). A advocacia da marca significa uma relação cimentada entre o consumidor e a marca, um relacionamento intenso com altos níveis de confiança e afeição e as comunidades de marca ajudarão a fortalecer essas relações. Nesse caso, e apoiado nas pesquisas de Lawer e Know (2006) e Carroll e Ahuvia (2006), propomos as seguintes hipóteses.

H13a: existe uma relação positiva entre a Comunidade de Marca (Dimensão Identificação) e a Advocacia da Marca.

H13b: existe uma relação positiva entre Comunidade de Marca (Dimensão Compromisso) e a Advocacia da Marca.

H16: existe uma relação positiva entre Amor à Marca e a Advocacia da Marca.

\subsubsection{Lealdade da marca}

A Lealdade à marca é definida como o suporte repetido e consistente a uma marca ao longo do tempo (BACK, 2005). A lealdade à marca é mais do que recompra, mas também uma relação psicológica de longo prazo (OLIVER, 1997). Clientes fiéis estão dispostos a pagar mais por um produto, até mesmo além de suas expectativas, pois confiam na marca e colocam os seus produtos e serviços em primeiro lugar, tendo menos intenção de deixar sua marca (ANDERSON; NARUS, 1990; BOWEN; CHEN, 2001; BOWEN; SHOEMAKER, 1998; LADHARI; BRUN; MORALES, 2008). O conceito de lealdade está fortemente relacionado com marketing de relacionamento, na medida em que é necessário desenvolver um relacionamento com a marca para que o indivíduo se torne fiel (SHETH, 1996). Antes de qualquer definição de estratégia, é necessário que as organizações conheçam seus clientes, as suas preferências e como eles podem executar melhor a sua estratégia (DICK; BASU, 1994). Segundo Kim et al. (2008), o compromisso que os membros de uma comunidade de marca demonstram com a sua marca leva à extrema lealdade. Indivíduos que fazem parte de uma comunidade de marca tendem a comprar essa marca consistentemente ao longo dos anos (ALGESHEIMER; DHOLAKIA; HERRMANN, 2005). Assim, as participações ativas em uma comunidade de marca, juntamente com suas interações com outros membros, ajudam a fortalecer a experiência da marca e aumentar seu valor para os consumidores (JANG et al., 2008). O envolvimento dos consumidores com uma comunidade de marca que leva a participar tem um efeito positivo nas vendas (ADJEI; NOBLE; NOBLE, 2010) e no comportamento do consumidor (BAGOZZI; DHOLAKIA, 2002) e, especialmente, na fidelidade à marca (MADUPU; COOLEY, 2010). As comunidades de marcas conseguem aumentar a lealdade dos consumidores à marca e causar oposição a outras concorrentes (LUEDICKE; THOMPSON; GIESLER, 2007; THOMPSON; SINHA, 2008). Portanto, as hipóteses formuladas são:

H14a: existe uma relação positiva entre a Comunidade de Marca (Dimensão Identificação) e a Lealdade à Marca.

H14b: existe uma relação positiva entre Comunidade de Marca (Dimensão Compromisso) e a Lealdade à Marca.

H17: existe uma relação positiva entre Amor à Marca e Lealdade à Marca. 
Figura 1 - Modelo Concetual

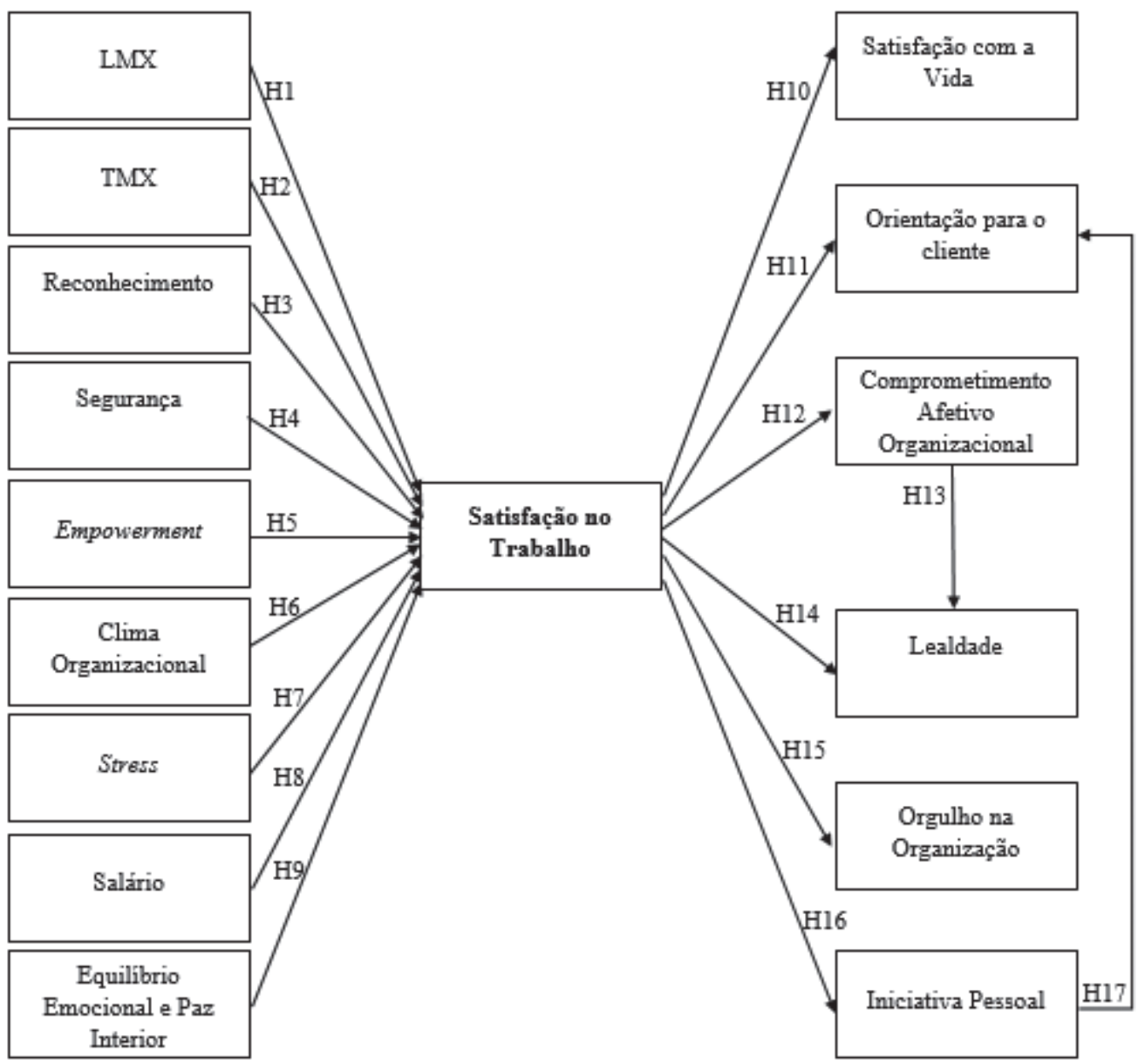

Fonte: (ALGESHEIMER; DHOLAKIA; HERRMANN, 2005).

\section{RECOLHA DE AMOSTRA E DADOS}

\subsection{SELEÇÃO DE AMOSTRA}

A população da amostra deste estudo é constituída por indivíduos do sexo feminino e masculino em território português, com vários grupos etários e diferenças entre os níveis de escolaridade e o rendimento do agregado familiar. Nenhum pré-requisito foi necessário para poder participar neste estudo. A técnica utilizada nos questionários foi a da bola de neve, sendo a amostra de natureza não probabilística. Para testar o modelo de investigação proposto e as hipóteses de pesquisa, os dados foram recolhidos com base em um questionário estrutura- do. Os questionários foram entregues a indivíduos com idade entre 18 e 90 anos, contatados nas ruas de duas cidades, autopreenchidos por eles. Assim, no total, 600 indivíduos foram contatados, e 510 concordaram em preencher o questionário. Ao longo do estudo, poderemos observar que existiu disparidade no que toca ao resultado deste estudo. Isso pode ser justificado pela forma ainda prematura como o povo português encara as comunidades de marca.

\subsection{PERFIL DA AMOSTRA}

A amostra é constituída por $58 \%$ do sexo feminino e $42 \%$ do sexo masculino, o que revela que ambos os sexos estão praticamente balanceados neste estudo. Cerca de 57,8\% com 
idade entre 18 e 30 anos, $29,6 \%$ entre 31 e 50 anos, $10,2 \%$ com idade entre 51 e 70 anos e $2,4 \%$ acima de 70 . Quanto aos níveis de escolaridade, $47,7 \%$ não são graduados e 53, 3\% são graduados. Em relação ao domicílio dos entrevistados, o maior percentual é de $29,8 \%$, sendo três pessoas no domicílio a resposta mais relatada. De acordo com a situação conjugal dos entrevistados, a maioria da amostra é solteira $(56,3 \%)$, seguida pelos casados ou casais residentes na mesma casa, com um percentual de $35,3 \%$. A maioria dos entrevistados, cerca de $48,2 \%$, são trabalhadores, seguidos dos indivíduos que ainda são estudantes, com $26,1 \%$ da amostra. Em relação à renda familiar, 41,4\% possuem renda inferior a 999 euros.

\subsection{MEDIDAS}

O formato da pesquisa consistia em perguntas de múltipla escolha, em que os indivíduos eram solicitados a responder ao que mais lhes convinha. A medida usada neste questionário foi baseada em uma escala Likert de sete pontos, variando de "Discordo fortemente" a "Concordo totalmente". As medidas para avaliar cada variável foram coletadas e adaptadas de investigações anteriores:

A necessidade de afiliação foi medida usando a escala de Baumeister e Leary (1995). Itens como "Ter amigos é muito importante" ou "Acho que qualquer experiência é mais significativa quando compartilhada com um amigo" foram usados.

A identificação foi medida usando a escala de Algesheimer, Dholakia e Herrmann (2005). Itens como "Estou muito próximo da comunidade" ou "Eu e os outros membros da Comunidade compartilhamos os mesmos objetivos" foram usados.

A extroversão foi medida usando a escala de Goldberg et al. (1997). Itens como "Eu vejo-me como alguém que ... é falador" ou "Eu vejo-me como alguém que tem uma personalidade assertiva".

A satisfação foi medida usando a escala de Russell-Bennett, McColl-Kennedy e Coote
(2007). Itens como "Estou satisfeito com minha decisão de comprar a marca X" ou "Eu sinto-me bem em considerar a Marca X minha marca favorita" foram usados.

A identificação da marca foi medida usando a escala de Kim, Han e Park, 2001 e Mael e Ashforth (1992). Itens como "Os sucessos da marca X são meus sucessos" ou "Quando alguém elogia a marca $X$, sinto que é um elogio pessoal" foram usados.

A confiança na marca foi medida usando a escala de Chaudhuri e Holbrook (2001). Itens como "Eu confio na Marca X." ou "A Marca X é segura" foram usados.

A satisfação com a relação foi medida pela escala de Jap (2011). Itens como "Estou satisfeito com meu relacionamento com essa marca" foram usados.

O prestígio da marca foi medido usando a escala de Stokburger-Sauer, Ratneshwar e Sen (2012). Itens como "A marca X é prestigiada" ou "Essa marca X é top com alta qualidade" foram usados.

A identidade de marca foi medida usando a escala de Bhattacharya e Sen (2003). Itens como "A marca X tem uma identidade distinta" ou "A marca X tem uma reputação elevada" foram usados.

$\mathrm{O}$ afeto da marca foi medido usando a escala de Thompson et al. (2005). Itens como "Marca X é carinhosa" ou "Marca X é amada" foram usados.

A comunidade de marca foi medida usando a escala de Algesheimer, Dholakia e Herrmann (2005) e Zhou et al. (2012), baseados em duas dimensões: identificação com a comunidade da marca e compromisso com a comunidade da marca. Itens como "Eu vejo-me como parte da comunidade de marca X" ou "Eu sentir-me-ia perdido se a comunidade de marca $\mathrm{X}$ não estivesse mais disponível" foram usados.

O passa-a-palavra foi medido usando a escala de Carroll e Ahuvia (2006). Itens como "Estou constantemente a fazer uma boa publicidade sobre a marca X" foram usados.

A advocacia da marca foi medida usando as métricas de Bendapudi e Berry (1997). A 
escala contém itens como "Eu defendo a marca $\mathrm{X}$ quando alguém diz algo negativo sobre isso" foram usados.

A lealdade à marca foi medida usando a escala usada por Carroll e Ahuvia (2006). Itens como "esta é a única marca que levarei em conta no futuro quando for comprar este tipo de produto" foram usados.

Amor à Marca foi medido com base na escala de Carroll e Ahuvia (2006) e Batra et al. (2012). Itens como "Estou apaixonado por esta marca" foram usados.

\subsection{VALIDADE}

Todos os itens foram medidos em uma escala Likert de sete pontos $(1=$ discordo totalmente a $7=$ concordo totalmente). A análise fatorial confirmatória foi utilizada para avaliar as propriedades psicométricas das escalas e o ajuste do modelo de mensuração, utilizando o AMOS 21. O modelo final mostra um bom ajuste (índice de ajustamento incremental = 0,957 ; índice tucker lewis $=0,949$; índice de ajuste comparativo $=0,956$; erro quadrático médio de aproximação $=0,044$, qui quadrado / grau de liberdade $=2,749)$. A confiabilidade composta e a variância média extraída foram calculadas. Todas as escalas apresentaram valores acima de 0,7 na variância média extraída, que estão em consonância com as recomendações (HAIR JUNIOR et al., 2006). A validade discriminante é evidenciada pelo fato de que todas as correlações entre os constructos são significativamente menores que 1 , e as correlações ao quadrado calculadas para cada par de constructos são sempre menores que a variância extraída para construções correspondentes (FORNELL; LARKER, 1981; SHIU et al., 2011), confirmando assim a validade discriminante (quadro n. 1).

Quadro 1 - Correlações, Alfa de Cronbach, Confiabilidade composta e Variância média extraída

\begin{tabular}{|l|l|l|l|l|l|l|l|l|l|l|l|l|l|l|l|l|l|l|}
\hline & DP & X1 & X2 & X3 & X4 & X5 & X6 & X7 & X8 & X9 & X10 & X11 & X12 & X13 & X14 & X15 & C.R. & AVE \\
\hline Necessidade de Afiliação (X1) & 0,881 & $\mathbf{0 , 7 7 8}$ & & & & & & & & & & & & & & & 0,787 & 0,553 \\
\hline Identificação (X2) & 1,716 & 0,012 & $\mathbf{0 , 9 3 9}$ & & & & & & & & & & & & & & 0,940 & 0,839 \\
\hline Extroversão (X3) & 1,189 & 0,207 & 0,116 & $\mathbf{0 , 8 6 0}$ & & & & & & & & & & & & & 0,866 & 0,621 \\
\hline Satisfação (X4) & 1,043 & 0,234 & 0,130 & 0,105 & $\mathbf{0 , 8 8 8}$ & & & & & & & & & & & & 0,898 & 0,748 \\
\hline Confiança na Marca (X5) & 0,964 & 0,252 & 0,130 & 0,125 & 0,720 & $\mathbf{0 , 8 7 8}$ & & & & & & & & & & & 0,883 & 0,716 \\
\hline Satisfação com o Relacionamento (X6) & 1,043 & 0,186 & 0,152 & 0,094 & 0,790 & 0,762 & $\mathbf{0 , 8 7 7}$ & & & & & & & & & & 0,880 & 0,710 \\
\hline Prestịio da Marca (X7) & 0,961 & 0.190 & 0,042 & 0,096 & 0,489 & 0,453 & 0.449 & $\mathbf{0 , 9 3 0}$ & & & & & & & & & 0,932 & 0,820 \\
\hline Identidade da Marca (X8) & 1,031 & 0,174 & 0,052 & 0,100 & 0,482 & 0,391 & 0,461 & 0,848 & $\mathbf{0 , 9 0 4}$ & & & & & & & & 0,905 & 0,761 \\
\hline Afeto da Marca (X9) & 1,109 & 0,138 & 0,176 & 0,096 & 0,412 & 0,370 & 0,446 & 0,382 & 0,446 & 0,876 & & & & & & & 0,877 & 0,704 \\
\hline Identifi. com a Comunidade de Marca (X10) & 1,850 & 0,014 & 0,852 & 0,125 & 0,137 & 0,132 & 0,181 & 0,030 & 0,040 & 0,165 & $\mathbf{0 , 9 3 4}$ & & & & & & 0,935 & 0,828 \\
\hline Comprom. com a Comunidade de Marca (X11) & 1,727 & 0,004 & 0,074 & 0,011 & 0,015 & 0,014 & 0,016 & 0,006 & 0,014 & 0,027 & 0,061 & $\mathbf{0 , 9 5 1}$ & & & & & 0,952 & 0,834 \\
\hline Amor à Marca (X12) & 0,896 & 0,136 & 0,217 & 0,110 & 0,666 & 0,552 & 0,591 & 0,342 & 0,359 & 0,456 & 0,228 & 0,028 & $\mathbf{0 , 8 5 2}$ & & & & 0,863 & 0,611 \\
\hline Passa-a-Palavra (WOM) (X13) & 1,611 & 0,096 & 0,420 & 0,167 & 0,403 & 0,358 & 0,377 & 0,155 & 0,160 & 0,277 & 0,420 & 0,035 & 0,366 & $\mathbf{0 , 8 7 1}$ & & & 0,880 & 0,710 \\
\hline Advocacia da Marca (X14) & 1,288 & 0,130 & 0,299 & 0,162 & 0,491 & 0,469 & 0,486 & 0,207 & 0,218 & 0,259 & 0,307 & 0,025 & 0,359 & 0,861 & $\mathbf{0 , 8 8 6}$ & & 0,887 & 0,724 \\
\hline Lealdade à Marca (X15) & 1,861 & 0,030 & 0,364 & 0,097 & 0,228 & 0,184 & 0,223 & 0,090 & 0,099 & 0,163 & 0,391 & 0,020 & 0,220 & 0,336 & 0,315 & $\mathbf{0 , 9 0 1}$ & 0,900 & 0,750 \\
\hline
\end{tabular}

Fonte: (HAIR JUNIOR et al., 2006).

\section{RESULTADOS E DISCUSSÃO}

Este estudo mostra que as comunidades de marcas têm um forte impacto no amor à marca, passa-a-palavra, advocacia e fidelidade à marca. Os resultados mostram também que 0 amor à marca revela um forte impacto nas variáveis positivas de passa-palavra, advocacia e fidelidade à marca. É agora importante discutir os resultados que provêm do teste de hipóteses apresentado anteriormente. Efetivamente, alcançar um senso de amor pela marca deve ser um dos objetivos críticos de qualquer empresa.

\subsection{ANÁLISE DE ANTECEDENTES DA COMUNIDADE DA MARCA}

É possível observar, no teste de hipótese, que a conexão entre o constructo necessidade de afiliação e as Comunidades de Marca, H1a e H2b, não é significativa em nenhuma de suas dimensões. Assim, não há evidências para concluir que a necessidade de afiliação aumenta a identificação ou comprometimento com a comunidade da marca. Olhando para a relação entre o constructo de identificação e a comunidade de marca, pode notar-se que é uma relação significativa para ambas as hipóteses, H2a e $\mathrm{H} 2 \mathrm{~b}$, corroborando a hipótese $\mathrm{H} 2$, que está 
em consonância com o paradigma de avaliação dessa variável. Em relação às relações entre a extroversão e a comunidade de marca, hipóteses $\mathrm{H} 3 \mathrm{a}$ e H3b, verifica-se que os resultados não coincidiram totalmente com os esperados. Notou-se que a relação entre a extroversão e a comunidade da marca foi significativa com a dimensão identificação, mas, ao contrário do esperado, a relação entre extroversão e comprometimento com a comunidade da marca não foi significativa e ainda exibiu uma relação negativa. No que diz respeito às ligações entre a satisfação e a comunidade da marca, H4a e H4b, os resultados são diferentes dos esperados. Pode observar-se que a relação entre a dimensão de identificação e a comunidade de marca não é significativa e que a ligação entre a satisfação e a dimensão do compromisso com a comunidade de marca também não existe, e ainda apresenta valores negativos. Não há como demonstrar que, quando a satisfação aumenta, também aumentam a identificação e o comprometimento com a comunidade. É possível observar que no que se refere às relações entre confiança da marca e comunidade da marca, ao contrário do que está descrito na literatura sobre o assunto, os resultados das hipóteses H6a e H6b não foram significativos. Há também uma relação negativa entre a confiança na marca e a identificação com a comunidade da marca, e não há dados estatísticos para apoiar essa relação. Observando a relação entre o constructo de satisfação de relacionamento e as comunidades de marca, $\mathrm{H} 7 \mathrm{a}$ e $\mathrm{H} 7 \mathrm{~b}$, pode ver-se que os resultados não coincidem exatamente com o que se esperava. Pode-se observar que a relação entre a satisfação com a relação com a identificação com a comunidade da marca é significativa, mas contrária às expectativas, a hipótese $\mathrm{H} 7 \mathrm{~b}$, o elo entre satisfação com o relacionamento e comprometimento com a comunidade da marca, não é significativa, mostrando sinal ainda negativo. Em relação aos vínculos entre prestígio de marca e comunidades de marca, nas hipóteses H8a e H8b, pode observar-se que nenhum foi considerado significativo, apresentando relações de negatividade nas duas dimensões da comunidade da marca. No que diz respeito às ligações entre a identidade da marca e a comunidade da marca, H9a e H9b, os resultados são diferentes dos esperados. Observa-se que a relação com a identificação da dimensão não foi significativa, mostrando-se ainda com valores negativos. Pode verificar-se que, em relação às relações entre o afeto da marca e a comunidade da marca, ao contrário do que está descrito na literatura sobre o assunto, os resultados das hipóteses H10a e H10b não foram significativos, e não há dados estatísticos que comprovem a autenticidade dessa relação.

\subsection{ANÁLISE DOS CONSEQUENTES DA COMUNIDADE DA MARCA}

Observando a relação entre o constructo da comunidade da marca e o amor à marca, H11a e H11b, pode observar-se que os resultados não coincidem completamente com o esperado. É possível observar que a dimensão de identificação da comunidade da marca tem um impacto positivo no amor à marca, mas esse impacto não ocorre para a dimensão do compromisso (H11b). Ainda assim, globalmente pode afirmar-se que existe uma relação significativa entre a comunidade da marca e o amor à marca. Em relação às relações entre a comunidade da marca $\mathrm{e}$ o passa-a-palavra positivo, nas hipóteses H12a e H12b, constata-se que os resultados não coincidem totalmente com o esperado. Nota-se que a relação entre a comunidade da marca e o passa-a-palavra positivo é significativa quando observamos a relação com a dimensão identificação. Contrariando as expectativas, a relação entre o comprometimento com a comunidade da marca e o passa-a-palavra positivo não se mostrou significativa, mostrando uma relação negativa. No entanto, pode dizer-se que existe uma relação significativa e que H12 é parcialmente corroborada. Quanto às ligações entre comunidade de marca e advocacia da marca, H13a e H13b, os resultados são diferentes do esperado. Pode ver-se que a relação com a dimensão de identificação foi significativa, mas que a dimensão compromisso não mostrou que existe relação 
significativa, sendo esta última de natureza negativa. No entanto, pode concluir-se que existe uma ligação significativa entre a comunidade de marca e a advocacia da marca, com $\mathrm{H} 13$ sendo parcialmente corroborada. Pode verificar-se que, em relação às relações entre a comunidade de marca e a fidelidade à marca, conforme descrito na literatura sobre o assunto, os resultados das hipóteses H14a e H14b mostraram-se um pouco diferentes do que seria esperado. É notável que exista uma relação significativa entre identificação e fidelidade à marca, mas, ao contrário das expectativas, a relação entre comprometimento e fidelidade à marca não é significativa e apresenta um sinal negativo. Consequentemente, pode afirmar-se que existe uma relação entre as comunidades de marca e a fidelidade à marca, e essa hipótese, H14, é parcialmente corroborada. Observando a relação entre o constructo de amor à marca e passa-a-palavra positivo, H15, os resultados coincidem completamente com o que se esperava. É claro que existe uma relação positiva entre o amor à marca e o passa-a-palavra positivo, corroborando a Hipótese H15, o que está de acordo com os estudos realizados por Carrol e Ahuvia (2006), bem como os estudos realizados por Batra et al. (2012). Em relação à conexão entre o constructo de amor à marca e a advocacia da marca, H16, pode-se dizer que os resultados coincidem totalmente com o que se esperava. Isso leva a essa hipótese, H16, sendo corroborada pelo estudo em questão, uma vez que os constructos se relacionam claramente entre si, o que está de acordo com os autores Carroll e Ahuvia (2006) e Batra et al. (2012). Quanto às ligações entre $\mathrm{o}$ amor à marca $\mathrm{e}$ a lealdade à marca, o H17, os resultados são claramente esperados e descritos na literatura existente sobre o assunto em questão. A lealdade leva a um relacionamento sério e compromisso com a marca (BACK, 2005; OLIVER, 1997; KIM et al., 2008; ALGESHEIMER; DHOLAKIA; HERRMANN, 2005). Pode observar-se que essas duas variáveis estão consistentemente relacionadas entre si, concluindo que a hipótese H17 é corroborada neste estudo.

Em relação aos antecedentes das comu- nidades de marcas, podemos destacar que a identificação, a extroversão e a satisfação com o relacionamento são, na verdade, variáveis consistentes quando se fala em antecedentes de comunidades de marcas. Esse histórico melhora não apenas o relacionamento dos consumidores com as marcas, mas também é uma ferramenta poderosa para as organizações. Finalmente, no que diz respeito às consequências das comunidades de marca, a importância de todos os constructos - Amor à Marca, Passa-A-Palavra Positivo, Advocacia da Marca e Lealdade à Marca - pode ser denotada por uma verdadeira relação entre as comunidades de marca e os seus consequentes, o que comprova a relevância deste empírica deste estudo.

\section{CONCLUSÕES}

Esta pesquisa teve como objetivo investigar as Comunidades de Marcas, identificando o seu impacto nas atitudes e comportamentos do consumidor, particularmente no amor à marca, passa-a-palavra, advocacia e lealdade à marca. Os resultados do estudo mostram que a dimensão de Identificação das Comunidades de Marca tem um efeito importante no amor à marca, no passa-a-palavra, advocacia e lealdade à marca. Contrariamente ao que era esperado, muitos dos antecedentes das Comunidades de Marca que foram propostos neste estudo não se mostraram relevantes. Isto pode ser explicado pelo facto de a amostra do estudo ser apenas o povo português. Ela revela que ainda não tem maturidade para se comprometer com uma comunidade de marca. No entanto, é revelado que, quando na amostra, ama uma marca, fala bem dessa mesma marca, assim como a defende e é leal a ela.

Esses resultados podem ser úteis para empresas que, possuindo uma marca já estável ou ainda em crescimento, querem que os seus consumidores sejam envolvidos no processo da sua expansão, e que os indivíduos sejam integrados à marca e se sintam parte dela. Isso é possível por meio das comunidades de marca. Os resultados também devem ser um estímulo 
para pesquisas na área de marketing de relacionamento e branding. Este estudo apresenta muitas pistas interessantes para pesquisa que constituem uma novidade significativa e explora as possibilidades introduzidas pelas comunidades de marca na construção do amor e lealdade à marca, como também na sua consolidação.

\section{CONTRIBUIÇÕES E LIMITAÇÕES}

\subsection{CONTRIBUIÇÕES TEÓRICAS}

Para McAlexander, Schouten e Koenig (2002), as comunidades de marcas são consideradas o santo graal da lealdade, que considera as comunidades, cada vez mais, importantes quando falamos de crescimento, não apenas na organização, mas também no crescimento global.

Quando se trata de comunidades de marca, é importante entender que não são apenas os consumidores que operam nelas, mas um número de entidades, incluindo as próprias empresas. É crucial perceber que conceitos semelhantes, como marketing de relacionamento e branding podem ser cobertos por essas comunidades, que são extremamente importantes hoje em dia. É necessário construir um relacionamento estável não apenas com os consumidores, mas também com todos os indivíduos que atuam no mercado e que estão, de algum modo, relacionados à marca. Mediante a comunidade da marca, é possível entender as necessidades dos consumidores, bem como o que pode ser necessário mudar. Comunidades de marca podem ser o meio para reforçar e estabilizar as relações entre a marca e os clientes.

Essa investigação inova estabelecendo uma relação empírica entre as comunidades de marcas e o amor à marca, que medeia as relações entre as comunidades de marca e os resultados relacionais, como passa-a-palavra positiva, advocacia e lealdade. Esta investigação ajuda a estabelecer a cadeia de efeitos entre o envolvimento da comunidade e os resultados relacionais, usando a mediação do amor à marca.

Por isso, e por se tratar de um estudo inovador na cultura portuguesa, é importante salientar que todas as suas contribuições se devem, em parte, a estudos anteriores, destacando os de McAlexander, Schouten e Koenig (2002), Muñiz Junior e O'Guinn (2001) e Carroll e Ahuvia (2006). Esses estudos testaram alguns dos antecedentes e consequências das relações com as comunidades da marca. Espera-se que ele possa contribuir para o enriquecimento teórico do tema apresentado. É ainda mais provável que o estudo contenha as informações necessárias para gerenciar melhor as marcas e os mercados.

\subsection{CONTRIBUIÇÕES PRÁTICAS}

A própria definição de comunidade de marca é baseada em um relacionamento especial entre o cliente e a marca, bem como entre o cliente e a organização a que a marca pertence e também entre o cliente e o produto em uso, bem como entre o outro consumidor, membros da mesma marca (MCALEXANDER; SCHOULTEN; KOENING, 2002). Dessa forma, as empresas devem olhar para as comunidades de marca como um fator altamente competitivo. É importante que as organizações estabeleçam e estimulem essas comunidades para que elas ajudem as marcas a crescer.

As marcas top-of-mind baseiam-se, essencialmente, na construção da mente que o consumidor faz sobre elas, o que significa que esse fenômeno aliado a uma comunidade forte pode manter essa marca no topo da mente do consumidor por anos. Ao estreitar as relações entre uma empresa e dos seus consumidores, é possível levar a marca a um nível superior: uma marca amada. Além disso, por meio de uma comunidade de marca, é fácil alcançar mais clientes e mais membros. Uma comunidade de marca pode reforçar os laços entre os membros, entre os membros e a marca, atrair novos membros e novos clientes e estabelecer relacionamentos duradouros, aumentando o passa-a-palavra positivo, advocacia e lealdade à marca. Essa investigação contém informações interessantes necessárias para gerenciar melhor as marcas e os mercados. 


\subsection{LIMITAÇÕES E PESQUISAS FU- TURAS}

Esta investigação baseia-se na coleta de dados transversais de uma amostra de consumidores portugueses. Quando relações causais devem ser exploradas, dados longitudinais são úteis para compreender questões de causalidade. Ao mesmo tempo, as escalas utilizadas, desenvolvidas e testadas em outros contextos podem não representar os sentimentos dos clientes portugueses.

Finalmente, a introdução de efeitos moderadores como gênero e idade, ou mesmo atitudes em relação a marcas e atitudes em relação ao dinheiro, pode contribuir para introduzir um contexto em que esses efeitos e impactos possam ser mais bem compreendidos.

\section{REFERÊNCIAS}

AAKER, D. A. Building strong brands. [S.l.]: Free Press, 1996.

ADJEI, M.; NOBLE, S.; NOBLE, C. The influence of $\mathrm{C} 2 \mathrm{C}$ communications in online brand communities on customer purchase behavior. Journal of the Academy of Marketing Science, v. 38, n. 5, p. 634-653, 2010.

AHUVIA, A. C. Beyond the extended self: loved objects and consumers' identity narratives. Journal of Consumer Research, v. 32, p. 171184, 2005.

ALEXANDER, R. S. Marketing Definitions. American Marketing Association, Chicago, 1964.

ALGESHEIMER, R.; DHOLAKIA, U. M.; HERMANN, A. The social influence of brand community: evidence from european car clubs. Journal of Marketing, v. 69, p. 19-34, 2005.

ALTMAN, I.; TAYLOR, D. A. Social penetration: The development of interpersonal relationships. Oxford, England: Holt, Rinehart \&
Winston, 1973.

ANDERSON, J. C.; NARUS, J. A. A model of distributor firm and manufacturer firm working partnerships. Journal of Marketing, v. 54, p. 42-58, 1990.

ARNDT, J. Role of product: related conversations in the diffusion of a new product. Journal of Marketing Research, v. 4, p. 291-295, 1967.

BACK, K. J. The effects of image congruence on customers brand loyalty in the upper middleclass hostel industry. Journal of Hospitality and Tourism Research, v. 29, n. 4, p. 448467, 2005.

BAGOZZI, R. P.; DHOLAKIAM, U. M. Intentional social action in virtual communities. Journal of Interactive Marketing, v. 16, p. 2-21, 2002.

BATRA, R. et al. Brand Love. Journal of Marketing, v. 76, n. 2, p. 1-16, 2012.

BAUMAN, Z. Comunidade: a busca por segurança no mundo atual. Rio de Janeiro: Jorge Zahar Editor, 2003.

BAUMEISTER, R. F.; LEARY, M. R. The need to belong: Desire for interpersonal attachments as a fundamental human motivation. Psychological Bulletin, v. 117, p. 497-529, 1995.

BELK, R. W. Possessions and the Extended Self. Journal of Consumer Research, v. 15, n. 2, p. 139-168, 1964.

BENDAPUDI, N.; BERRY, L. L. Customers' motivations for maintaining relationships with service providers. Journal of Retailing, v. 73, p. 15-37, 1997.

BERGAMI, M.; BAGOZZI, R. P. Self-Categorization, affective commitment and group self-esteem as distinct aspects of social identity 
in the organization. British Journal of Social Psychology, v. 39, p. 555-577, 2000.

BHATTACHARYA, C.; SEN, S. Consumer-company identification: a framework for understanding consumers' relationships with companies. Journal of Marketing, v. 67, p. 76-88, 2003.

BOWEN, J. T.; CHEN, S. L. The relationship between customer loyalty and customer satisfaction. International Journal of Contemporary Hospitality Management, v. 13, n. 5, p. 213-217, 2001.

BOWEN, J. T.; SHOEMAKER, S. Loyalty: a strategic commitment. Cornell Hotel and Restaurant Administration Quarterly, v. 39, p. 12-25, 1998.

BOWER, G. H.; FORGAS, J. P. Affect, memory, and social cognition. In: EICH, E. et al. Niedenthal, Cognition and emotion. New York, NY, US: Oxford University Press, 2000. p. $87-168$.

BUTTLE, F. A. Word of mouth: understanding and managing referral marketing. Journal of Strategic Marketing, v. 6, p. 241-254, 1998.

CABLE, D. M.; GINO, F.; STAATS, B. R. Breaking them in or eliciting their best? Reframing socialization around newcomers' authentic self-expression". Administrative Science Quarterly, v. 58, n. 1, p. 1-36, 2013.

CARROLL, B. A.; AHUVIA, A. C. Some antecedents and outcomes of brand love. Market Lett, v. 17, p. 79-89, 2006.

CHAUDHURI, A.; HOLBROOK, M. B. The chain of effects from brand trust and brand affect to brand performance: the role of brand loyalty. Journal of Marketing, v. 65, n. 2, p. 81-93, 2001.

CHERNATONY, L. Consumer-based brand equity conceptualization and measurement a literature review. International Journal of Market Research, v. 52, n. 1, 2010.

DICK, A. S.; BASU, K. Customer loyalty: toward an integrated conceptual framework. Journal of the Academy of Marketing Science, v. 22, p. 99-113, 1994.

DHOLAKIAA, U. M.; BAGOZZIA, R. P.; PEARO, L. K. A social influence model of consumer participation in network and smallgroup-based virtual communities. Intern. J. of Research in Marketing, v. 21, p. 241-263, 2004.

ESCALAS, J. E.; BETTMAN, J.R.; Self-construal, reference groups, and brand meaning. Journal of Consumer Research, v. 32, p. 378389, 2005.

FORNELL, C.; LARCKER, D. F. Evaluating structural equation models with unobservable variables and measurement error. Journal of Marketing Research, v. 18, n. 1, p. 39-50, 1981.

FOURNIER, S. Consumers and their brands: developing relationship theory in consumer research. The Journal of Consumer Research, v. 24, n. 4, p. 343-373, 1998.

GOLDBERG, D. P. et al. The validity of two versions of GHQ in the WHO study of mental illness in general health care. Psychological Medicine, v. 27, p. 191-197, 1997.

GRAY, J. A. The psychophysiological basis of introversion-extraversion. Behavior Research and Therapy, v. 8, n. 3, p. 249-266, 1970.

GUMMESSON, E. Relationship marketing and a new economy: it's time for de-programming. Journal of Services Marketing, v. 16, n. 7, p. $585-589,2002$.

HAIR, JUNIOR, J. F. et al. Multivariate Data 
Analysis. 6. ed. Upper Saddle River, NJ: Pearson Prentice Hall, 2006.

HERR, P. et al. Effects of word-of-mouth and product-attribute information on persuasion: an accessibility-diagnosticity perspective. Journal of Consumer Research, v. 17, n. 4, p. 454$62,1991$.

HILL, C. A. Affiliation motivation: People who need people... but in different ways. Journal of Personality and Social Psychology, v. 52, n. 5, p. 1008-1018, 1987.

HOGG, M. A. Social Identity Theory. In: Burke, P. J. (ed.). Contemporary social psychological theories. [S.l.]: Stanford University Press, 2006. p. 111-136.

HUNG, Hsiu-Yu. Attachment, identification, and loyalty: examinating mediating mechanisms across brand and brand community contexts. Journal of Brand Management, v. 21, n. 7/8, p. 594-614, 2014.

JANG, H. et al. The influence of on-line brand community characteristics on community commitment and brand loyalty. International Journal of Electronic Commerce, v. 12, p. 57-80, 2008.

JAP. Self-esteem and life satisfaction as mediators between parental bonding and psychological well-being in Japanese young adults. International Journal of Psychology and Counselling, v. 3, n. 1, p. 1-8, 2011.

JONES, R.; KIM, Y. K. Single-brand retailers: building brand loyalty in the off-line environment. Journal of Retailing and Consumer Services, v. 18, n. 4, p. 333-340, 2011.

KAPFERER, J. N. The new strategic brand management: creating and sustaining brand equity long term. Kogan Page Publishers, p. 5-557, 2008.
KEH, H. T. et al. The effects of entrepreneurial orientation and marketing information on the performance of SMEs. Journal of Business Venturing, v. 22, p. 592-611, 2007.

KEMPF, D. S. Attitude formation from product trial: Distinct roles of cognition and affect for hedonic and functional products. Psychology and Marketing, v. 16, p. 35-50, 1999.

KIM, C. K.; HAN, D.; PARK, S. The effect of brand personality and brand identification on brand loyalty: applying the theory of social identification. Japanese Psychological Research, v. 43, n. 4, p. 195-206, 2001.

KIM, J.; MORRIS, J. D. The power of affective response and cognitive structure in product-trial attitude formation. Journal of Advertising, v. 36, n. 1, p. 95-106, 2007.

KIM, D. J. et al. A Trust-based consumer decision-making model in electronic commerce: the role of trust, perceived risk, and their antecedents. Decision Support Systems, v. 44, n. 2, p. 544-564, 2008.

KUENZEL, S.; HALLIDAY, S. V. Investing antecedents and consequences of brand identification. Journal of Product \& Brand Management, v. 17, n. 5, p. 293-304, 2008.

LADHARI, R.; BRUN, I.; MORALES, M. Determinants of dining satisfaction and post-dining behavioral intentions. International Journal of Hospitality Management, v. 27, n. 4, p. 563-573, 2008.

LARASATI, Y.; HANANTO, A. The role of value congruity and consumer brand identification toward development of brand commitment and positive word of mouth. Department of Management, Universitas Indonesia, 2012.

LAROCHE, M. et al. The effects of social media based brand communities on brand community markers, value creation practices, brand 
trust and brand loyalty. Computers in Human Behavior, n. 28, p. 1755-1767, 2012.

LAWER, C.; KNOX, S. Customer advocacy and brand development. Journal of Product \& Brand Management, v. 15/2, p. 121-129, 2006.

LUCAS, R. E. et al. Factors influencing the relation between extraversion and pleasant affect. Personality and Social Psychology, v. 79, n. 6, p. 1039-1056, 2000.

LUEDICKE, M. K.; THOMPSON, C. J.; GIESLER, M. consumer identity work as moral protagonism: how myth and ideology animate a brand-mediated moral conflict. Journal of Consumer Research, v. 36, n. 6, p. 10161032, 2007.

MADUPU, V.; COOLEY, D. O. Antecedents and consequences of online brand community participation: a conceptual framework. Journal of Internet Commerce, v. 9, n. 2, p. 127$147,2010$.

MAEL, F.; ASHFORTH, B. E. Alumni and their alma mater: a partial test of the reformulated model of organizational identification. Journal of Organizational Behavior, v. 13, p. 103-123, 1992.

MAISAM, Shirkhodaie; MAHSA, Rastgoo-deylami. positive word of mouth marketing: explaining the roles of value congruity and brand love. Journal of Competitiveness, v. 8, n. 1, p. 19-37, 2016.

MATTSSON, L. G. "Relationship marketing" and the "markets-as-networks approach"- a comparative analysis of two evolving streams of research. Journal of Marketing Management, v. 5, p. 447-461, 1997.

MCALEXANDER, J. H.; SCHOUTEN, J. W.; KOENIG, H. F. Building brand community. Journal of Marketing, v. 66, p. 38-54, 2002.
MCCARTHY, E. J.; PERREAULT, W. D. Basic marketing: a managerial approach. Homewood, Ill.: Irwin, 1987.

MILLÁN, Ángel; DÍAZ, Estrella. Analysis of consumers' response to brand community integration and brand identification. Journal of Brand Management, v. 21, n. 3, p. 254-272, 2014.

MITTAL, V.; KAMAKURA, W. A. Satisfaction, repurchase intent, and repurchase behavior: investigating the moderating effect of customer characteristics. Journal of Marketing Research, v. 38, n. 1, p. 131-142, 2001.

MORGAN, R. M.; HUNT, S. D. The Commitment-trust theory of relationship marketing. Journal of Marketing, v. 58, p. 20-38, 1994.

MUNIZ JUNIOR, A. M.; O'GUINN, T.C. Brand Community. Journal of Consumer Research, v. 27, p. 412-432, 2001.

OLIVER, R. L. Satisfaction: A behavioral perspective on the consumer. New York: The McGraw-Hill Companies, Inc., 1997.

PARK et al. Being immersed in social networking environment: facebook groups, uses and gratifications, and social outcomes. $\mathbf{C y}$ berpsychology $\&$ behavior: the impact of the Internet, multimedia and virtual reality on behavior and society, v. 12, n. 6, p. 729-33, 2009.

PARK. C. W.; MACINNIS, D. J. Beyond attitudes: attachment and consumer behavior. Seoul Journal of Business, v. 12, p. 3-35, 2006.

RUSSELL-BENNETT; McCOLL-KENNEDY, J. R.; COOTE, L. V. Involvement, satisfaction, and brand loyalty in a small business services setting. Journal of Business Research, v. 60 , n. 12, p. 1253-1260, 2007. 
SCARPI, D. Does size matter? An examination of small and large web-based brand communities. Journal of Interactive Marketing, v. 24, n. 1, p. 14-21, 2010.

SCHAU, H. J.; MUNIZ, A. M.; ARNOULD, E. J. How Brand community practices create value. Journal of Marketing, v. 73, p. 30-51, 2009.

SHAARI, Hasnizam; AHMAD, Intan S. Brand evangelism among online brand community members. International Review of Management and Business Research, v. 5, n 1, p. 80$88,2016$.

SHAVER, P.; BUHRMESTER, D. Loneliness, sex role orientation and group life: social needs perspective. Baulus, P.B., Ed., Basic Group Processes, Springer-Verlag, New York, p. 259-288, 1983.

SHETH, J. N. Word-of-mouth in low-risk innovations. Journal of Advertising Research, v. 11, p. 15-18, 1971.

SHETH, J. N. Organizational buying behavior: past performance and future expectations. Journal of Business \& Industrial Marketing, v. 11, n. 3/4, p. 7-24, 1996.

SHIU, E. et al. Reflections on discriminant validity: reexamining the bove et al. findings. Journal of Business Research, v. 64, n. 5, p. 497-500, 2011.

SIRGY, M. J. Self-concept in consumer behavior: a critical review. Journal of Consumer Research, v. 9, p. 287-300, 1982.

STEENKAMP, J-B. et al. How perceived brand globalness creates brand value. Journal of International Business Studies, v. 34, n. 1, p. 53-65, 2003.

STOKBURGER-SAUER, N.; RATNESHWARB, S.; SEN, S. Drivers of consumer-brand identification. Forthcoming. International Journal of Research in Marketing, p. 1-55, 2012.

SWEENEY, J. C.; SOUTAR, G. N. Consumer perceived value: the development of a multiple item scale. Journal of Retailing, v. 77, n. 2, p. 203-220, 2001.

THOMPSON, M. et al. The ties that bind: measuring the strength of consumers' emotional attachments to brands. Journal of Consumer Psychology, v. 15, n. 1, p. 77-91, 2005.

THOMPSON, S. A.; SINHA, R. K. Brand communities and new product adoption: the influence and limits of oppositional loyalty. Journal of Marketing, v. 72, p. 65-80, 2008.

TSAI, H.; HUANG, H.; CHIU, Y. Brand community participation in taiwan: examining the roles of individual-, group-, and relationship-level antecedents. Journal of Business Research, v. 65, p. 676-684, 2012.

TSE, D. K.; WILTON, P. C. Models of consumer satisfaction formation: an extension. Journal of Marketing Research, v. 25, n. 2, p. 204-212, 1988.

WALZ, A.; CELUCH, K. The effect of retailer communication on customer advocacy: The moderating role of trust. Journal of Consumer Satisfaction, Dissatisfaction and Complaining Behavior, v. 23, p. 95-110, 2010.

WANG, H. J. Heteroscedasticity and non-monotonic efficiency effects of a stochastic frontier model. Journal of Productivity Analysis, v. 18, n. 3, p. 241-253, 2002.

WASKO, M. M. et al. Collective action and knowledge contribution in electronic networks of practice. Journal of the Association for Information Systems, v. 5, n. 11, article 15, 2004. 
WEBSTER, F. E. The changing role of marketing in the corporation. Journal of Marketing, v. 56 , p. 1-17, 1992.

WEISS, R. S. Loneliness: the experience of emotional and social isolation. Cambridge, MA: MIT Press, 1973.

ZHOU, Z. et al. How do brand communities generate brand relationships? Intermediate mechanisms. Journal of Business Research, n. 65, p. 890-895, 2012.

ZIAULLAH, M. et al. Exploring the relationship between justice and supply chain process integration through linkage of trust-an empirical study of pakistan. Research in Business and Management, v. 2, n. 1, 2015. 